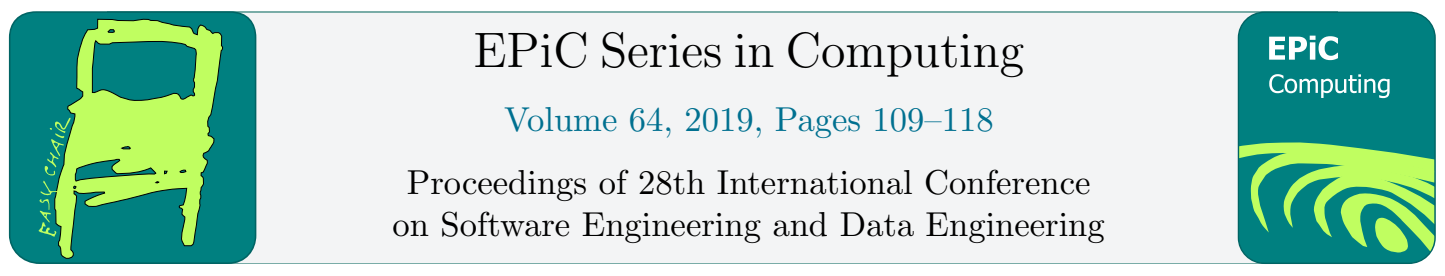

\title{
Evaluation of Mobile Augmented Reality Application for Building Evacuation
}

\author{
James Stigall ${ }^{1}$ and Sharad Sharma ${ }^{2}$ \\ Department of Computer Science \\ Bowie State University, Bowie, MD, USA \\ ${ }^{1}$ stigallj0813@students.bowiestate.edu, ${ }^{2}$ ssharma@bowiestate.edu
}

\begin{abstract}
Building occupants must know how to properly exit a building should the need ever arise. Being aware of appropriate evacuation procedures eliminates (or reduces) the risk of injury and death occurring during an existing catastrophe. Augmented reality (AR) is increasingly being sought after as a teaching and training tool because it offers a visualization and interaction capability that captures the learner's attention and enhances the learner's capacity to retain what was learned. Utilizing the visualization and interaction capability that AR offers and the need for emergency evacuation training, this paper explores mobile AR application (MARA) constructed to help users evacuate a building in the event of an emergency such as a building fire, active shooter, earthquake, and similar circumstances. The MARA was built for Android-based devices using Unity and Vuforia. Its features include the use of intelligent signs (i.e. visual cues to guide users to the exits) to help users evacuate a building. Inter alia, this paper discusses the MARA's implementation and its evaluation through a user study utilizing the Technology Acceptance Model (TAM) and the System Usability Scale (SUS) frameworks. The results demonstrate the participants' opinions that the MARA is both usable and effective in helping users evacuate a building.
\end{abstract}

\section{Introduction}

To curb the number of injuries and fatalities suffered during an emergency such as a building fire, active shooter, or an earthquake, it is critical that practical emergency evacuation plans are in place. The goal of creating an emergency evacuation plan is to ensure that occupants vacate the premises as quickly as possible [1]. Emergency evacuation plans should not only consider the path taken to safety but also other factors such as equipment needed and disabled occupants [2]. Also, communication about the nature and extent of the emergencies must consider individual evacuee behavior, as evacuees may either not take evacuation orders seriously enough or may experience excessive degrees of panic during the evacuation process [3]. Conducting evacuation drills is a quintessential method for preparing 
building patrons to evacuate a building in case of an emergency [4]. However, drill participants may lack the time or physical ability to participate such drills. Augmented reality (AR) is an emerging technology not only used for entertainment purposes (e.g. gaming and social media), but also for teaching and instruction. It is manifested as computer-generated objects juxtaposed upon the real (i.e. actual) environment. This creates a learning environment that fosters imagination and immersion where the user can interact with those objects in real time [5],[6]. It is being employed to teach a wide variety of subjects from English to anatomy to car assembly [7]-[9].

For these reasons, a mobile augmented reality application (MARA) was developed to help users evacuate a building in case of an emergency (that is, an unanticipated, albeit foreseeable, event). The application was developed using the Unity game engine and the Vuforia AR Toolkit. The MARA features 3D models of the Computer Science Building at Bowie State University which were modelled in SketchUp. This research is motivated by previous work reported in [10] and is an extension of the work noted in [11]. This paper is further organized as follows: Section 2 discusses other works related to the subject MARA presented herein; Section 3 details the design and implementation of the MARA; Section 4 provides the evaluation framework upon which the user study for the MARA is based; Section 5 discusses the user study in detail; Section 6 articulates the results and implications of the user study; and Section 7 suggests areas for future work.

\section{Related Work}

\subsection{Mobile AR}

Tsunezaki et al [12] have proposed a mobile AR application that mimicked the material of real objects. The proposed system intakes (using the camera on a device on which the application has previously been installed) the pose of the user's hand and regenerates the shape and reflectance of a real object as a 3D virtual object. The virtual object, after it has been generated, can be translated and rotated using the user's hand. Tsunkezaki et al captured images of real objects (the target objects) using a Microsoft Kinect connected to a PC. The Kinect captured the images in a dark room using only a small LED light as the only light source so that surrounding light would not affect the reflection of the target object captured.

Hoang et al. [13] proposed a passive deformable haptic glove which was incorporated with a Digital Foam sensor as virtual material overlaid on a physical object to allow the user to cut into and extrude from the object's surface. When the user wears the glove, it captures the force applied by the user's hand when the user touches real objects. With this system, the user cuts a pre-defined shape by pressing the glove against a physical surface or dragging it across the surface. The glove tracks the pose of the user's hand using six degrees of freedom and uses one additional degree of freedom for the surface depth information. That information is then juxtaposed on top of the physical surface being modified. The goal of the system proposed in [13] is to reduce error and fatigue.

Waechter et al. [14] discuss an application that captures the movement of people in real-time. The application utilizes a ceiling camera that captures a bird's eye view of the multiple people occupying the area around it. The application uses image processing techniques to distinguish people from each other and from other objects. To use this application, a user points the device camera towards a marker. Once the marker is detected, the user's location information is estimated and is linked with the positions of the people tracked by the application. The device with the application installed connects to a central server using a Wi-Fi connection to send the user's position information and request the positions of the other people tracked by the application. Other works on mobile augmented reality include a service oriented framework for a GPS application proposed by Shah and Agrawal [15] and a teaching module that aims to increase the user's learnability when using the module to learn a particular subject [16]. 


\subsection{AR for Evacuation and Emergency Management}

An application developed for HoloLens, a head-mounted display, helping users evacuate a building was discussed by Stigall et al. in [17]. The application generated 3D floorplans along with the path to the nearest exit and a marker showing the user's location. A user study evaluating that application concluded that it was useful for training purposes and served as an ideal substitute for 2D evacuation plans. Weding and Parent [18] have proposed an AR system which allows emergency personnel to view, in real time, parts of a building via drones to determine evacuation paths for people or pets trapped in the building, and so on. In that system, emergency personnel sent drones to desired locations throughout the building. In turn, the drones sent back videos and images with sensor information (from smoke sensors) and other data augmented within the drones. Emergency personnel viewed the videos and images from a computer screen and used them to determine a plan of action to rescue trapped occupants.

\section{Design and Implementation of the MARA}

The development of the present MARA employed the waterfall model, a six stage software development process where each stage is planned in advance. The six stages are requirements definition, analysis, design, implementation, testing, and maintenance. The first four stages of the model (requirements definition, analysis, design, and implementation) are discussed in this section, while the testing stage is discussed in Sections 4-6, addressing the user study. Finally, the maintenance stage is discussed with the conclusion and future work in Section 7.

\subsection{Requirements Definition Stage}

Two sets of requirements were necessary as a part of the MARA's development: functional requirements to address system executions and nonfunctional requirements to address user expectations. The functional requirements for the MARA are as follows:

- When the user points the device camera towards the target image, the floorplan for that target image should be generated atop the image.

- In order to avoid information overload, the user should be able to either show or hide the intelligent signs using the toggle buttons placed on the screen.

- The MARA should facilitate quick and effective evacuation.

- The MARA should be simple to operate.

- The user should be able to ascertain the appropriate path to evacuation from the user's location.

- The avatars should guide the user to the exit.

- The exits should be clearly marked.

The MARA's nonfunctional requirements are as follows:

- The executable (.apk) file should not be too large for the user's device.

- The user interface should be intuitive: easy to grasp.

- The MARA should not lag nor should it crash.

- The MARA should be updated as needed.

\subsection{Analysis Stage}

The analysis phase consisted of, first, obtaining floorplans of the Computer Science Building. Once the floorplans were obtained, they were modelled in 3D space using SketchUp. The details of each floor 
(i.e. walls, doors, and windows) were drawn in the same manner so that he floorplans appeared as they would be seen in real life.

\subsubsection{Intelligent Signs}

The authors define as intelligent signs as those visual cues in the MARA which guide users to the exits. Four intelligent signs were identified to guide the user out of the building by indicating the location of each exit and the path to arrive at each exit. These intelligent signs included:

1. Blinking exit signs

2. Moving green doors

3. Blue arrows

4. Picture hints

The intelligent signs were incorporated into the floorplan so that they appeared on the floorplan when it is generated on the screen. The intelligent signs can be seen in Figure 1. These intelligent signs and their functions are discussed in Section 3.4.

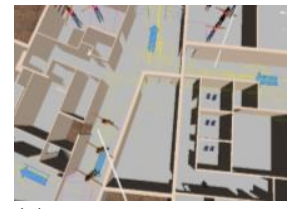

(a)

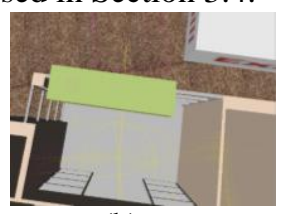

(b)

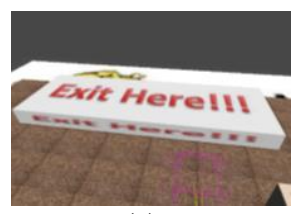

(c)

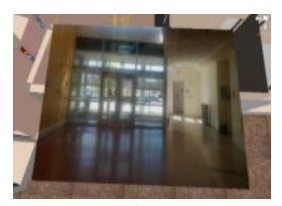

(d)

Figure 1: The MARA's intelligent signs: (a) blue arrows, (b) moving green doors, (c) exit signs, and (d) picture hints. See Fig. 4, infra.

\subsection{Design Stage}

The MARA's design follows the composite design pattern where software components are organized into parent-child hierarchies. The MARA's design pattern is shown in Figure 2. In the composite design pattern, the markers act as the parent to the floorplan (represented by the "floor" component in the diagram) and the floorplan act as the parent to the virtual fire and smoke, avatars, and intelligent signs. Further, each avatar contains two components of its own - the speed at which it walks towards the exit (i.e. "Speed" in Figure 2) and the path it takes to get to that exit.

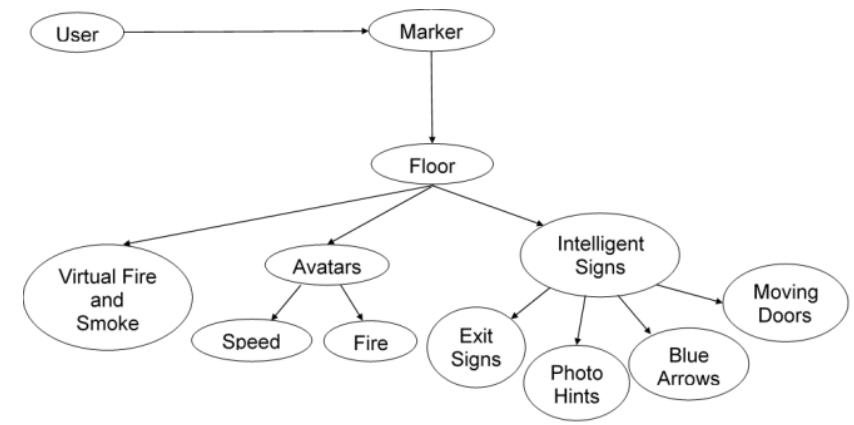

Figure 2: The MARA's software design pattern.

\subsection{Implementation Stage}

Implementation of the MARA was comprised of three stages. In the first phase of the MARA's implementation, the three floorplans were drawn in SketchUp according to the 2D floorplans obtained. 
They were drawn to appear as realistic as possible - to look the same as they would in real life. Objects such as desks, tables, computers, and chairs were added on each floor. The floorplans were saved as SketchUp (.skp) files and exported from SketchUp to 3dsMax for the next phase.

In the second phase, the .skp files were imported into 3dsMax. There, extraneous objects were deleted so that the files corresponding to the models outputted from this phase would not be too big to be used in Unity in the third phase. Once that was done, the models were saved as .obj files so that they could be exported from 3dsMax to Unity for the next phase.

In the third phase, the .obj files were imported into Unity where the MARA would be built. All of the three floorplans were placed in a Unity scene. Within each floor, avatars were added and were assigned both a path to follow to get to the exit and speeds at which to traverse this path. Additionally, virtual fire and smoke were placed on each floor to add a sense of urgency when the user sees the floor on his or her screen. The intelligent signs were implemented on each floor as follows:

- The blinking exit signs were 3D cubes placed at the exits on each floor. A texture with big red letters reading "Exit Here!!" was wrapped around each cube. Finally, animation was added to the blinking exit signs to make them move up and down on a continuous loop.

- The moving green doors were green 3D cubes placed wherever the doors are on the floors. Animations were given to them to make them move from left to right on a continuous loop.

- The blue arrows were 2D quad shapes with a blue arrow texture wrapped around them to make them appear as blue arrows. They were placed on the floors to guide the user to the exits.

- The photo hints were 2D quad shapes with textures wrapped around them. The textures were pictures taken of particular locations around the building. The photo hints were placed on the floors in relation to real locations of the pictures (e.g. the photo hint for the first floor snack machine was placed were the first floor snack machine is in real life).

The MARA uses three types of image markers (see Figure 3): (1) Type A to generate either one of the three floors in the building, (2) Type B to generate a floor with the specific location indicated by a red square, and (3) Type $\mathrm{C}$ to generate floor information when triggered by the marker. In Unity, each of the floors were placed atop their corresponding marker so that when the camera detects those markers, the appropriate floor can be seen. Now constructed, the MARA was packaged into an .apk file from Unity and transferred onto a mobile device. Figure 4 shows the MARA's user interface as it appears on the android Samsung tablet.
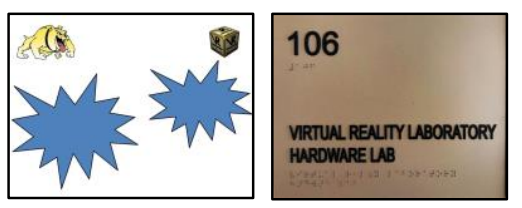

Figure 3: Markers: (a) Type A, (b) Type B, and (c) Type C.

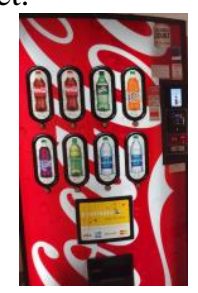

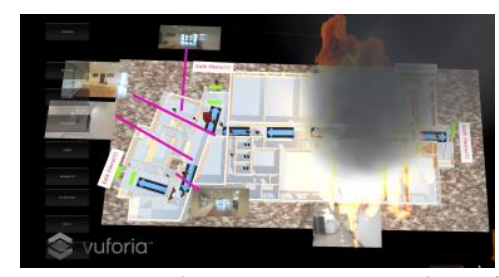

Figure 4: The MARA's user interface.

\section{Evaluation Frameworks}

A user study described more fully below was utilized to evaluate the MARA. The evaluation of the MARA was based on the SUS and the TAM frameworks. The questions from those frameworks were modified and used in the questionnaire for the user study. This section discusses the SUS and the TAM in further detail. 


\subsection{Technology Acceptance Model (TAM)}

In his evaluation, Davis [5] used a total of twelve Likert-scale items to evaluate CHART-MASTER. The first set of six items addressed the users' perception of usefulness towards CHART-MASTER and the second set of six items addressed the users' perception of ease of use towards CHART-MASTER. A seven-point scale was used to rate each item on Davis's evaluation. The lowest end of the scale represented "likely", the highest-end of the scale represented "unlikely", and the center of the scale represents a neutral attitude regarding the system [19].

\subsection{System Usability Scale (SUS)}

The framework uses 10 Likert scale questions. The responses to which can range from 1 to 5 with 1 meaning "strongly disagree" and 5 meaning "strongly agree". Responses are given a score contribution ranging from 0 to 4 and tallied to acquire a SUS score between 0 and 100 . The calculation of the SUS score for a given system is as follows:

1. For the odd-numbered questions (questions 1, 3, 5, 7, and 9), subtract one from the score contributions.

2. For the even-numbered questions (questions 2, 4, 6, 8, and 10), subtract five from the score contributions.

3. Total all of the score contributions, then multiply the sum by 2.5 [20].

\section{User Study}

\subsection{Purpose of the User Study}

The goal of the user study was to assess the usability and effectiveness of the MARA in helping people evacuate a building. The methodology entailed collecting data for users of different ages and backgrounds who participated in the user study.

\subsection{Hardware Used}

There were two types of devices used in this research: tablet and mobile phone. Two tablets were utilized: an ASUS Transformer Pad and the Samsung Galaxy Tab. Both devices ran the Android operating system. The ASUS ran Android 4.2.1 (the Jelly Bean release) while the Samsung ran Android 4.4 (the KitKat release). The screen resolution of the ASUS was 1920x1200 pixels and the screen resolution of the Samsung was 2560x1600 pixels. The mobile phone used in this research was the LG G7 ThinQ. It ran the Oreo release of the Android operating system (Android version 8.1) and its screen resolution was $3120 \times 1440$ pixels.

\subsection{Participants}

The user study had a total of 69 participants. Males constituted $46 \%$ of participants while $54 \%$ of the participants were females. Students (freshmen, sophomores, juniors, seniors, and graduate students) made up the bulk of the participants in the user study (85\%, to be exact) while the other $15 \%$ were staff, faculty, and administration (see Figure 5). The participants were broken down into two groups - insiders and outsiders. Insiders were the participants who regularly attended classes or performed work inside the Computer Science Building and the outsiders were the participants that did not regularly attend classes or performed work inside the Building. The insiders made up 55\% of the participants while the outsiders made up $45 \%$ of the participants. In the user study each session took 10-15 minutes. All 
participants were at least 18 years of age. Upon arrival, the participants underwent consent procedures in which the study personnel explained the study procedures and how to navigate and use the buttons in the MARA.

\subsection{MARA Evacuation Procedure}

As mentioned earlier, four different types of markers were used - three corresponding to each floor of the Computer Science Building and one showing the first floor but with a specific location indicated. There were two setups: in the first setup, each participant hovered a mobile phone in front of the markers; in the second setup, each participant hovered a tablet in front of the markers. When the participant hovered either the mobile phone or tablet in front of a marker, the appropriate floorplan was generated. At that time, the participant was given the option of using the toggle buttons on the user interface and view the intelligent signs along with the floorplan. After participating in the study, the users were given a questionnaire via Google Forms that described the ease or difficulty of their user experience. The results of the questionnaire are described in the next section.

\section{Data Implications: Results}

\subsection{Survey Questions \& Data}

The questionnaire featured questions from the TAM and the SUS frameworks modified for this user study. The modified SUS and TAM questions can be seen in Tables 1 and 2, respectively. Additional questions regarding the MARA's effectiveness and user demographics were also asked in the questionnaire. Similar to the original SUS questions, the SUS questions in the user study utilized the five-point scale for responses with 1 meaning "strongly disagree" and 5 meaning "strongly agree". Likewise, the TAM questions in the user study utilized the same seven-point scale for responses with 1 meaning "likely" and 7 meaning "unlikely". The first six questions of the TAM framework addressed the perceived usefulness of the MARA while the other six questions addressed the perceived ease-ofuse of the MARA. Regarding experience with using mobile applications, most of the participants (97\%) had, at least, limited experience (see Figure 6).

\begin{tabular}{|c|c|c|}
\hline & Question & $\begin{array}{c}\text { Qualitative Response (Av. } \\
\text { or Mean) }\end{array}$ \\
\hline 1) & I think that I would like to use the MARA frequently. & Somewhat Agree (3.5) \\
\hline 2) & $\begin{array}{l}\text { I found the use of the toggle buttons and the intelligent signs (i.e. exit signs, blue } \\
\text { arrows, moving green doors, and pictures) to be unnecessarily complex. }\end{array}$ & Neutral (2.9) \\
\hline 3) & $\begin{array}{l}\text { I thought the MARA's toggle buttons were easy to use (i.e. avatars, blue arrows, } \\
\text { moving green doors, pictures, and fire and smoke). }\end{array}$ & Somewhat Agree (3.9) \\
\hline 4) & $\begin{array}{l}\text { I think that I would need the support of a technical person to be able to use the } \\
\text { MARA. }\end{array}$ & Somewhat Disagree (2.3) \\
\hline 5) & $\begin{array}{l}\text { I found the following features in the MARA to be well-integrated: (1) intelligent signs } \\
\text { (exit signs, blue arrows, moving green doors, and pictures), (2) avatar animation, (3) } \\
\text { location marker (red square), (4) fire and smoke, and (5) toggle buttons). }\end{array}$ & Somewhat Agree (3.9) \\
\hline 6) & I thought that the directions to the nearest exit were not correct (inconsistent). & Somewhat Disagree (2.3) \\
\hline 7) & I would imagine that most people would learn to use the MARA very quickly. & Somewhat Agree (4.1) \\
\hline 8) & I found the MARA very cumbersome to use. & Neutral (2.9) \\
\hline 9) & I felt very confident using the MARA for instructional and educational purposes. & Somewhat Agree (3.9) \\
\hline 10) & $\begin{array}{l}\text { I needed to learn a lot of things before I could use the MARA (e.g. which buttons to } \\
\text { tap on, which toggle buttons to press, how to zoom in and out of floorplans, and } \\
\text { installing the application). }\end{array}$ & Somewhat Disagree (2.6) \\
\hline
\end{tabular}

Table 1: The SUS questions used in the user study. 


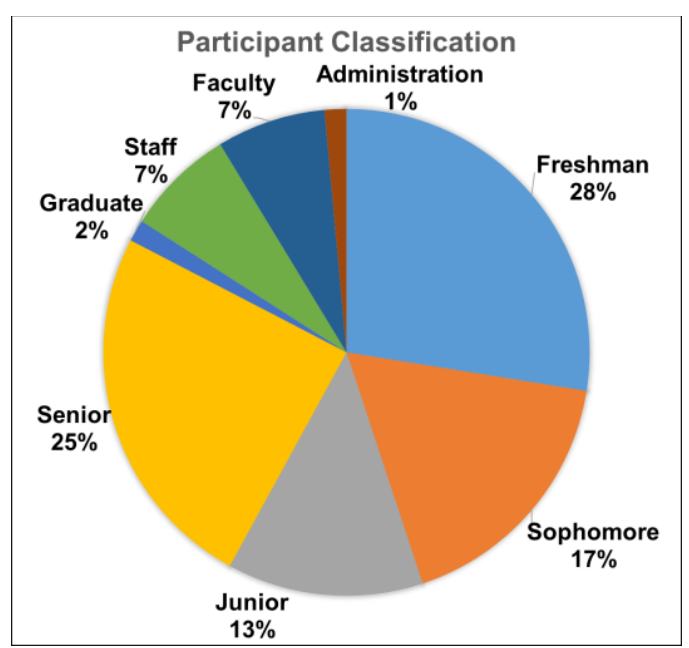

Figure 5: Participant classification.

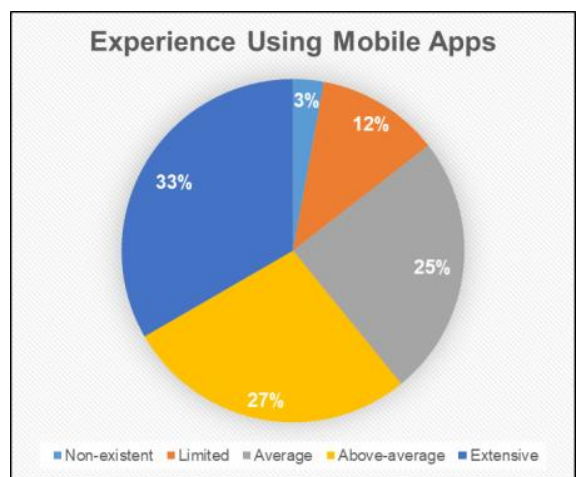

Figure 6: Participants' level of experience using mobile apps.

\subsection{Data Implications}

Bangor et al [21] have correlated SUS scores with the collegiate grading scale and with acceptability (a term indicating whether or not a system is "acceptable" or not) and adjective ratings ("best imaginable", "excellent", "good", "OK", "poor", "awful" and "worst imaginable"). The SUS scores were calculated for each response as described in Section 4.2. All of those scores were averaged to get 65.61594 as the average SUS score. According to the scale given in [21], the MARA described in this paper is marginally acceptable with an adjective rating of "OK" considering that the average SUS score for the MARA is 65.61594. The numerical responses for the TAM questions were also averaged. The qualitative value (e.g. "Likely", "Somewhat Likely", "Somewhat Unlikely", and "Unlikely") for each question can be seen in Table 2 along with the average numerical response for that question in parenthesis. The averages range from approximately 2.6 to approximately 2.9. Considering that 1 on the TAM scale corresponds to "Likely" and 7 on the scale corresponds to "Unlikely", the response averages correspond to "somewhat likely" suggesting that the users found the MARA useful and easyto-use.

Similar to the TAM and SUS questions, the numerical responses to the questions specific to the MARA were averaged (except for the last question). The averages for the first three of that set of questions correspond to "neutral" on the scale for those questions (i.e. the averages fall in the middle of the scale). This means that users, on average, had neutral opinions about those particular questions. Thus, there is a possibility that users would use the MARA during the emergency in place of a 2D evacuation plan and that it would improve their ability to exit the building in case of an emergency. To further demonstrate the MARA's usability, the results for questions most directly eliciting perceptions of ease and usability were selected from the questionnaire for further computation. Not only were the average responses for those questions were calculated, but also were the mode (the most popular) and the median (the middle value). Also, the responses to those questions indicate that users, typically, were able to operate the MARA and easily able to get acclimated to using it. This suggests that participants felt the MARA offered significant usability. 


\begin{tabular}{|ll|c|}
\hline \multicolumn{1}{|c|}{ Question } & Qualitative Response (Av. or Mean) \\
\hline 1) & Using the MARA would enable me to be more knowledgeable of exit signs. & Somewhat Likely (2.78) \\
\hline 2) & Using the MARA would improve my ability to evacuate the building safely. & Somewhat Likely (2.84) \\
\hline 3) & Using the MARA would help me evacuate the building quickly. & Somewhat Likely (2.9) \\
\hline 4$)$ & $\begin{array}{l}\text { Using the MARA would enhance my effectiveness in getting out of the } \\
\text { building. }\end{array}$ & Somewhat Likely (2.82) \\
\hline 5) & Using the MARA would make it easier to evacuate the building. & Somewhat Likely (2.87) \\
\hline 6) & I would find the MARA useful for evacuating the building. & Somewhat Likely (2.75) \\
\hline 7) & Learning to operate the MARA was easy for me. & Somewhat Likely (2.71) \\
\hline 8) & I found it easy to get the MARA to do what I want to do. & Somewhat Likely (2.75) \\
\hline 9) & My interaction with the MARA was clear and understandable. & Somewhat Likely (2.71) \\
\hline 10) & I found the MARA flexible to interact with. & Somewhat Likely (2.77) \\
\hline 11) & It would be easy for me to become skillful at using the MARA. & Somewhat Likely (2.62) \\
\hline 12) & I would find the MARA easy to use. & Somewhat Likely (2.61) \\
\hline
\end{tabular}

Table 2: Questionnaire results for the TAM.

\section{Conclusions and Future Work}

This paper proposed a MARA developed for Android to help users evacuate a building. It featured intelligent signs to guide users to the appropriate exits. It also featured 3D floorplans of the Computer Science Building which were drawn in SketchUp and were imported into Unity. Marker detection capabilities was implemented through the use of Unity and Vuforia. The application was evaluated through a user study where 69 participants used it and then answered a questionnaire based on the SUS and TAM frameworks. According to the results of the study, the users concluded that the MARA developed was indeed usable and that it was helpful for safe evacuation. It is suggested that future work include additional testing to validate the MARA, by the developers and by potential users; including furniture (e.g. tables, desks, chairs, and audio-visual equipment) within each floorplan and seeing what impact it would have on the users' evacuation; determining how applicable regulatory rules and procedures and compliance with such would affect the requirements definition; including open-ended questions to allow user study participants to identify factors which they felt rendered the MARA more (or less) usable; analyzing how specific populations responded to the questionnaire (e.g. students, staff, faculty, administration, technical majors, non-technical majors, experienced mobile app users and nonexperienced mobile app users); and developing the MARA to be compatible with other platforms such as the iPhone and Windows.

\section{References}

[1] M. Yusoff, J. Ariffin, and A. Mohamed, "Optimization Approaches for Macroscopic Emergency Evacuation Planning: A Survey" in 2008 International Symposium on Information Technology, Kuala Lumpur, Malaysia, Aug. 26-29, 2008.

[2] J. Pandolfo, "Top Five School Evacuation Lessons Learned", Education Digest, pp. 58-60, vol. 81, no. 7, 2016.

[3] R. Alsnih and P. Stopher, "A Review of the Procedures Associated with Devising Emergency Evacuation Plans", 2004.

[4] J. Pandolfo, "Best practices for LTC facility emergency evacuation", Long-Term Living, pp. 36$37,2015$. 
[5] S. Vassigh, A. Elias, F.R. Ortega, D. Davis, G. Gallardo, H. Alhaffar, L. Borges, J. Bernal, N.D. Rishe, "Integrating Building Information Modelling with Augmented Reality for Interdisciplinary Learning" in 2016 IEEE International Symposium on Mixed and Augmented Reality Adjunct Proceedings, Merida, Mexico, pp. 260-261, Sept. 19-23, 2016.

[6] A. Iriarte-Solis, P. González-Villegas, R. Fuentes-Covarrubias, and G. Fuentes-Covarrubias, "Mobile Guide to Augmented Reality for Campus of the Autonomous University of Nayarit" in 2016 IEEE International Symposium on Mixed and Augmented Reality Adjuct Proceedings, Merida, Mexico, pp. 1-4, Sept. 19-23, 2016.

[7] C.S.C. Dalim, T. Piumsomboon, A. Dey, M. Billinghurst, S. Sunar, "TeachAR: An Interactive Augmented Reality Tool for Teaching Basic English to Non-Native Children" in 2016 IEEE International Symposium on Mixed and Augmented Reality Adjunct Proceedings, Merida, Mexico, Sept. 19-23, 2016.

[8] T. Blum, V. Kleeberger, C. Bichlmeier, and N. Navab, "mirracle: An Augmented Reality Magic Mirror System for Anatomy" in 2012 IEEE Virtual Reality Workshops (VRW), Costa Mesa, CA, pp. 115-116, Mar. 4-8, 2012.

[9] R. Woll, T. Damerau, K. Wrasse, R. Stark, "Augmented reality in a serious game for manual assembly processes" in IEEE International Symposium on Mixed and Augmented Reality 2011, pp. 37-39, Basel, Switzerland, Oct. 26-29, 2011.

[10] S. Sharma and S. Jerripothula, "An Indoor Augmented Reality Mobile Application for Simulation Building Evacuation”, Proceedings of SPIE-IS\&T Electronic Imaging, vol. 9392, 2015.

[11] J. Stigall and S. Sharma, "Mobile Augmented Reality Application for Building Evacuation Using Intelligent Signs" in $26^{\text {th }}$ International Conference on Software Engineering and Data Engineering, San Diego, CA, Oct. 2-4, 2017.

[12] S. Tsunezaki, R. Nomura, T. Komuro, S. Yamamoto, and N. Tsumura, "Reproducing Material Appearance of Real Objects using Mobile Augmented Reality" in 2018 IEEE International Symposium on Mixed and Augmented Reality (ISMAR), Munich, Germany, pp. 196-197, Oct. 1620, 2018.

[13] T.N. Hoang, R.T. Smith, B.H. Thomas, "Passive Deformable Haptic Glove to Support 3D Interactions In Mobile Augmented Reality Environments" in IEEE International Symposium on Mixed and Augmented Reality 2013, Adelaide, Australia, pp. 257-258, Oct. 1-4, 2013.

[14]C.A.L. Waechter, D. Pustka, G.J. Klinker, "Vision based People Tracking for Ubiquitous Augmented Reality Applications" in IEEE International Symposium on Mixed and Augmented Reality 2009, Orlando, FL, USA, pp. 221-222, Oct. 19-22, 2009.

[15] J. Shah and B. Agrawal, "Prototype Algorithm Design for Web Based Service Oriented Architecture Mobile Augmented Reality System" in 2015 Fifth International Conference on Communication Systems and Network Technologies, Gwalior, India, pp. 1085-1088, Apr. 4-6, 2015.

[16]M. Durairaj and P.S. Aurelia, "A Modernistic and Contemporary Mobile Augmented Reality Erudition System" in Proceedings of the IEEE 2017 International Conference on Computing Methodologies and Communication, Erode, India, pp. 1109-1112, July 18-19, 2017.

[17] J. Stigall, S.T. Bodempudi, S. Sharma, D. Scribner, J. Grynovicki, and P. Grazaitis, "Building Evacuation using Microsoft HoloLens" in $27^{\text {th }}$ International Conference on Software Engineering and Data Engineering, New Orleans, LA, Oct. 8-10, 2018.

[18] K. Wedig and D. Parent, 'Augmented Reality of a Building', United States Patent, Jun. 26, 2018.

[19] M. Chuttur, "Overview of the Technology Acceptance Model: Origins, Developments and Future Directions", Sprouts: Working Papers on Information Systems, vol. 9, no. 37, 2009.

[20] J.R. Lewis and J. Sauro, "The Factor Structure of the System Usability Scale", in Human Centered Design, M. Kurosu, Ed. Springer-Verlag Berlin Heidelberg, pp. 94-103, 2009.

[21] A. Bangor, P. Kortum, and J. Miller, "Determining What Individual SUS Scores Mean: Adding an Adjective Rating Scale", Journal of Usability Studies, 4(3): 114-123, 2009. 